\title{
RIMAPS Characterization of Etch Pits on Deformed Aluminum Surfaces
}

\author{
E. A. Favret, and N. O. Fuentes \\ Comisión Nacional de Energía Atómica (CNEA), CAC, U.A. Materiales. Av. del Libertador 8250, \\ 1429 - Buenos Aires. Argentina. \\ Instituto de Tecnología "Prof. Jorge A. Sábato", Universidad Nacional de Gral. San Martín. \\ Av. Gral Paz 1499, B1650KNA - San Martín. Buenos Aires. Argentina.
}

In the present research work we analyze the surface of different crystalline grains of aluminum, which have been mechanically ground in one direction and chemically etched with Tucker reagent, by means of Rotated Image with Maximun Average Power Spectrum (RIMAPS) technique [1] [2]. When metals and alloys are etched with certain reagents, attack takes place at isolated points within the grains rather than by general surface etching. This localized etching is commonly known as etch-pits, which are linear-sided figures. In most cases these pits are formed by attack along definite crystallographic planes. The positions of the sides or faces of etch-pits are related to the orientations of the grains on which they are formed. Etch-pits are likely to form at regions of high dislocation density. The distribution of etch-figures on the surface of any given crystal is not random, it is directly related to its structure [3] [4].

In this work, the aluminum samples were annealed during 30 hours $\left(550^{\circ} \mathrm{C}\right)$, so that the grains in the sample would achieved the size of approximately $5 \mathrm{~mm}$. The crystals were orientated following the Back Laue Reflection method. All the sample were mechanically ground with emery paper down to 1000 grit. The chemical reagent used was: $\mathrm{HCl} 45 \mathrm{ml}, \mathrm{HNO}_{3} 15 \mathrm{ml}, \mathrm{HF} 15 \mathrm{ml}, \mathrm{H}_{2} \mathrm{O} 25 \mathrm{ml}$ [5]. The images used for RIMAPS analysis were obtained with scanning electron microscope (SEM) (see figures 1 and 2). The RIMAPS spectra of grains (174) and (323) are presented in figure 3. The existence of equal number of peaks in both spectra indicates that the typical etch-figure characterizing the damage on surfaces is the same: a triangle. On the other hand, the relative angular differences between peaks are not coincident. This is a direct consequence of been observing different crystallographic planes. It can be calculated from Laue information, the theoretical values of internal angles of the etched triangle. Comparison of these values with the angular differences given by RIMAPS, emphasizes the fact that RIMAPS determines not only the orientations that are in perfect accordance to the theoretical values, but also gives notice of the existence of surface deformation with the appearance of peaks denoting secondary directions, different from that theoretically expected.

\section{References}

[1] N. O. Fuentes and E. A. Favret, Journal of Microscopy. 206 (2002) 72.

[2] N. O. Fuentes and E. A. Favret, Microscopy and Analysis. 56 (2002) 5.

[3] E. A. Favret et al., Practical Metallography. 36 (1999) 206.

[4] G.E.G.Tucker and P.C. Murphy, J. Inst. Metals. 8 (1952-53) 235.

[5] P.Lacombe and L. Beaujard, J. Inst. Metals. 74 (1948) 1.

[6] G. F. Vander Voort, Metallography, Principles and Practice, McGraw Hill, New York, 1984. 


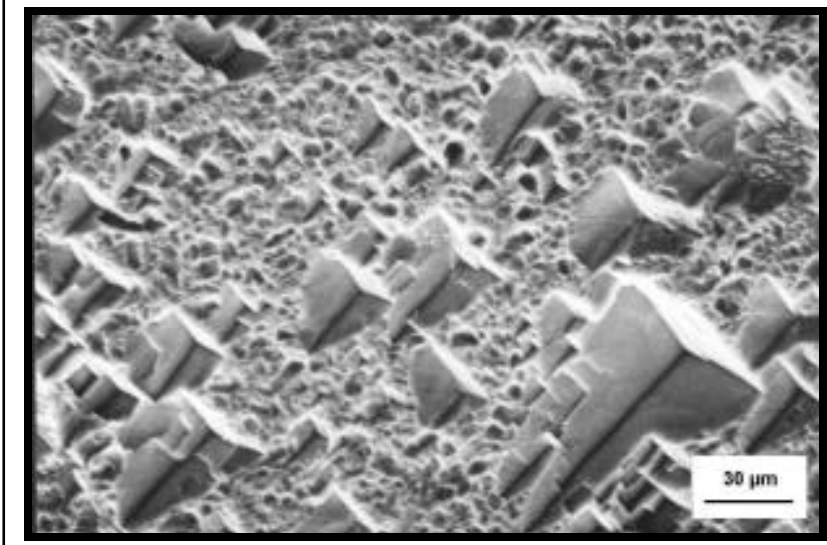

Figure 1. SEM micrograph of plane (174).

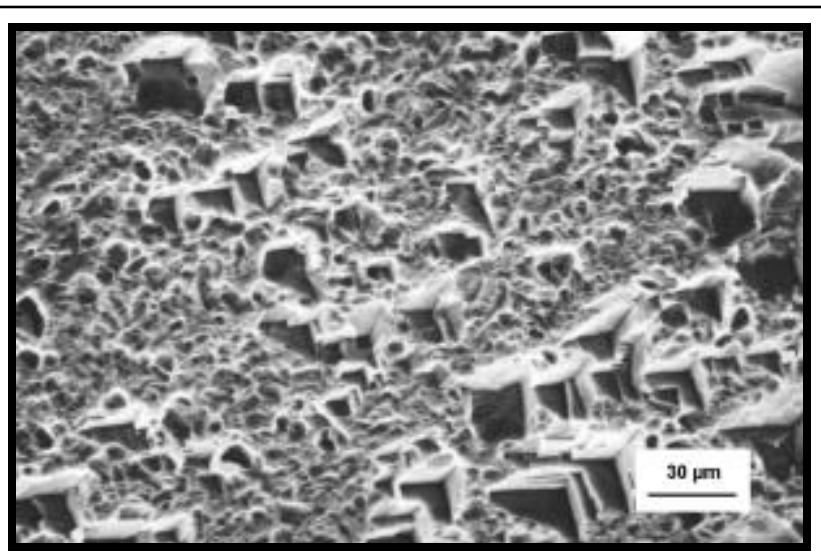

Figure 2. SEM micrograph of plane (323).

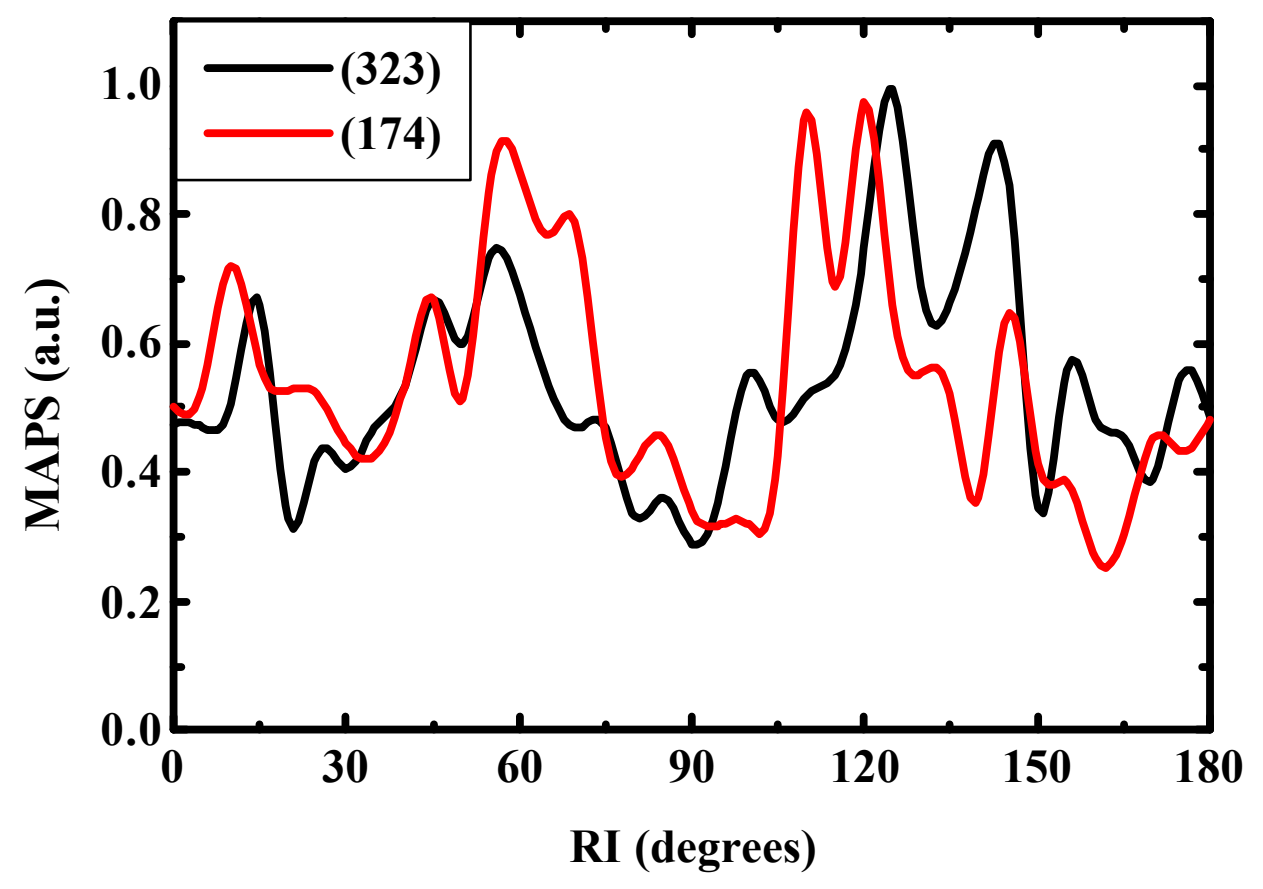

Figure 3: RIMAPS spectra of aluminum surfaces corresponding to crystallographic planes (174) and (323). 